\title{
Surface Modification of SUS 316L Stainless Steel with Tartaric Acid Derivative-Crosslinked Human Serum Albumin Matrices
}

\author{
Sachiro Kakinoki ${ }^{1}$, Yasuyuki Katada ${ }^{2}$, Yoshiyuki Uchida ${ }^{1}$, Tetsushi Taguchi ${ }^{1, *}$ \\ ${ }^{1}$ Biomaterials Center, National Institute for Materials Science, 1-1 Namiki, Tsukuba, Ibaraki 305-0044, Japan and \\ ${ }^{2}$ Materials Manufacturing and Engineering Station, National Institute for Materials Science, 1-2-1 Sengen, Tsukuba, \\ Ibaraki 305-0047, Japan
}

\begin{abstract}
The surface of stainless steel (SUS316L) was modified by alternating immersion in a solution of human serum albumin (HSA) and solution of a tartaric acid derivative (TAD). The resulting HSA/TAD-immobilized SUS316L was characterized by means of contact-angle measurement, attenuated total-reflectance Fourier-transform infrared spectroscopy, atomic force microscopy, and X-ray photoelectron spectroscopy. A HSA/TAD layer was formed on the surface of SUS316L, the thickness of which increased with increasing numbers of cycles of alternating immersion in the two solutions. The HSA/TAD layer on SUS316L was stable to washing in $1 \mathrm{M} \mathrm{NaCl}$ or 5 vol\% sodium dodecyl sulfate, showing that the layer was immobilized by covalent bonding rather than electrostatic or hydrophobic interaction. The presence of the HSA/TAD layer on the SUS316L suppressed the formation of a fibrin network. Alternating immersion in solutions of HSA and TAD is a useful technique for functionalizing the surfaces of metals.
\end{abstract}

\section{INTRODUCTION}

Stainless steel is widely used as a material for orthopedic implants and biomedical devices, such as bone plates, spinal rods, and coronary stents, owing to its corrosion resistance and superior mechanical properties [1]. Although stainless steel implants are generally biocompatibile, as are chromium and titanium alloys, problems frequently arise from biological reactions of the human body against the implanted metallic material. Once a metallic implant is brought into contact with the blood, protective responses of the human body induce adsorption of proteins and adhesion and activation of platelets. These protective responses trigger the formation of thrombi and lead to failure of the metallic implant [2]. Researchers have therefore been attempting to prepare biocompatible surfaces by chemical modification of metallic materials. A typical approach to the modification of a metal surface is to apply a coating that is chemically or physically adsorbed. Such coatings can be applied, for example, by layerby-layer (LbL) assembly technique [3, 4]. The LbL technique is based on the consecutive adsorption of polyanions and polycations through electrostatic interaction. Brynda et al. prepared ultrathin films of albumin and heparin as a blood anticoagulant device by means of this technique [5]. Although the electrostatic attraction between negatively and positively charged molecules confers a high selectivity and operability, LbL multilayers are generally unstable compared with chemically immobilized layers. Another approach to the modification of metal surface is by chemical immobilization of biofunctional molecules [6-9]. For example, Yoshioka et al. covalently immobilized alginic acid on the surface of stainless steel by using carbodiimide as a condensation reagent in order to confer blood compatibility [10]. Similarly,

*Address correspondence to this author at the Biomaterials Center, National Institute for Materials Science, 1-1 Namiki, Tsukuba, Ibaraki 305-0044, Japan; E-mail: TAGUCHI.Tetsushi@nims.go.jp
Müller et al. immobilized collagen on stainless steel to give a cell-compatible surface [11]. In most experiments, immobilization is carried out by using a coupling or crosslinking reagent, such as carbodiimide or glutaraldehyde, on an aminosilanized surface after physiochemical adsorption of the biopolymer, resulting in the formation of a monolayer of biopolymer on the stainless steel surface. These thin monolayers of biopolymers are effective for the modification of the stainless steel surfaces, but in some cases, a dense layer is required for use as a drug carrier to contain high doses of a drug within its matrix.

We have recently developed novel organic acid derivatives that contain succinimidyl ester moieties within their molecules for the preparation of novel biocompatible matrices [12-15]. Furthermore, we have developed a novel tissue adhesive that uses such an organic acid derivative as a crosslinker and have demonstrated that this adhesive shows an excellent biocompatibility and a high bonding strength $[16,17]$.

In the present study, we modified the surface of stainless steel by covalent immobilization of human serum albumin (HSA) by using a silane coupling agent and a tartaric acid derivative (TAD). The immobilized HSA/TAD multilayer was produced by alternating immersion in solutions of HSA and TAD. We expected that a covalently immobilized HSA/TAD multilayer on stainless steel would be more stable than films bound by intermolecular interactions such as electrostatic or hydrophobic interactions. Therefore, it believes that the stainless steel modified with HSA/TAD multilayer possesses the ability of suppressing adsorption of blood platelet and negative-charged proteins, and blood compatibility superior to surfaces modified with noncovalent bonds. Here, we characterized the resulting surface-modified stainless steel by means of contact-angle measurements, attenuated total reflectance Fourier-transform infrared spectroscopy (ATR-FTIR), atomic force microscopy (AFM), and X- 
ray photoelectron spectroscopy (XPS). The blood compatibility of HSA/TAD-coated stainless steel was also examined by using murine arterial blood.

\section{EXPERIMENTAL}

\section{Materials}

Stainless steel plates (AISI SUS316L) in the form of disks $10 \mathrm{~mm}$ in diameter and $1.0 \mathrm{~mm}$ thick were purchased from Rare Metallic Co., Ltd (Tokyo, Japan). (3-Aminopropyl) (trimethoxy)silane ( $\gamma$-APS), sodium chloride $(\mathrm{NaCl})$, sodium dodecyl sulfate (SDS) and $10 \%$ formalin neutral buffer solution ( $\mathrm{pH}$ 7.4) were purchased from Wako Pure Chemical Industries, Ltd. (Osaka, JAPAN). Tartaric acid, $N$ hydroxysuccinimide (HOSu) and doxorubicin hydrochloride (DOX) were from Wako Pure Chemical Industries, Ltd. (Osaka, JAPAN). Dicyclohexylcarbodiimide (DCC) was purchased from Kokusan Chemical Co., Ltd. (Tokyo, JAPAN). Human serum albumin (HSA) was purchased from Sigma-Aldrich Co. (St. Louis, MO, USA). All other reagents were used without further purification.

\section{Silanization of the SUS316L Surface}

The SUS316 plate was ground, mirror polished, and cleaned ultrasonically in acetone, ethyl acetate, and methanol: this is referred to as the bare metal plate. It was then immersed in $1 \mathrm{wt} \%$ aqueous $\mathrm{NaOH}$ solution for 1 min to form a basic oxidized surface, washed with distilled water, and dried with nitrogen gas. The plate was aminosilanized by treatment with $2 \mathrm{wt} \%$ aqueous $\gamma$-APS solution for $6 \mathrm{~h}$ in a nitrogen atmosphere. The aminosilanized SUS316L (abbreviated as AS) was washed ultrasonically in distilled water and then dried with nitrogen gas.

\section{Immobilization of HSA on Aminosilanized SUS316L}

HSA was immobilized on the AS plate by alternating immersion of the plate in a solution of TAD in DMSO and in an aqueous solution of HSA (Fig. 1). To activate the amino groups on the AS surface, the plate was first immersed in $200 \mathrm{mM}$ TAD/DMSO solution for $10 \mathrm{~min}$. The activated AS plate was washed well with DMSO and $\mathrm{MeOH}$, and then dried with nitrogen gas. It was then immersed in $0.5 \mathrm{wt} \%$ aqueous HSA solution for 10 min to immobilize HSA on the AS surface. The plate was finally washed with distilled water. This process, i.e. activation and immobilization of HSA, was repeated 5,10 , or 20 times (HSA/TAD-5, HSA/TAD10 , and HSA/TAD-20) to form a layer of the HSA/TAD matrix on the SUS316L surface (Fig. 2).

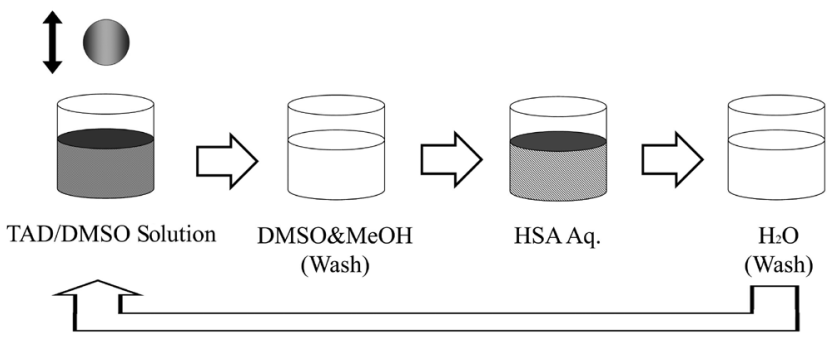

Fig. (1). Covalent surface modification of SUS316L by alternating immersion in HSA and TAD solutions.

\section{Surface Characterization}

The surfaces of samples were characterized by means of contact-angle measurement, ATR-FTIR, AFM, and XPS. The contact-angle with distilled water was measured by using a contact-angle meter (CA-W; Kyowa Interface Science Co., Ltd., Saitama, Japan). Images of the water spreading on the sample were recorded by a camera and analyzed by using FAMAS software (Kyowa Interface Science Co., Ltd., Saitama, Japan). Three samples were measured for each group. ATR-FTIR analysis of surface-modified SUS316L was per-
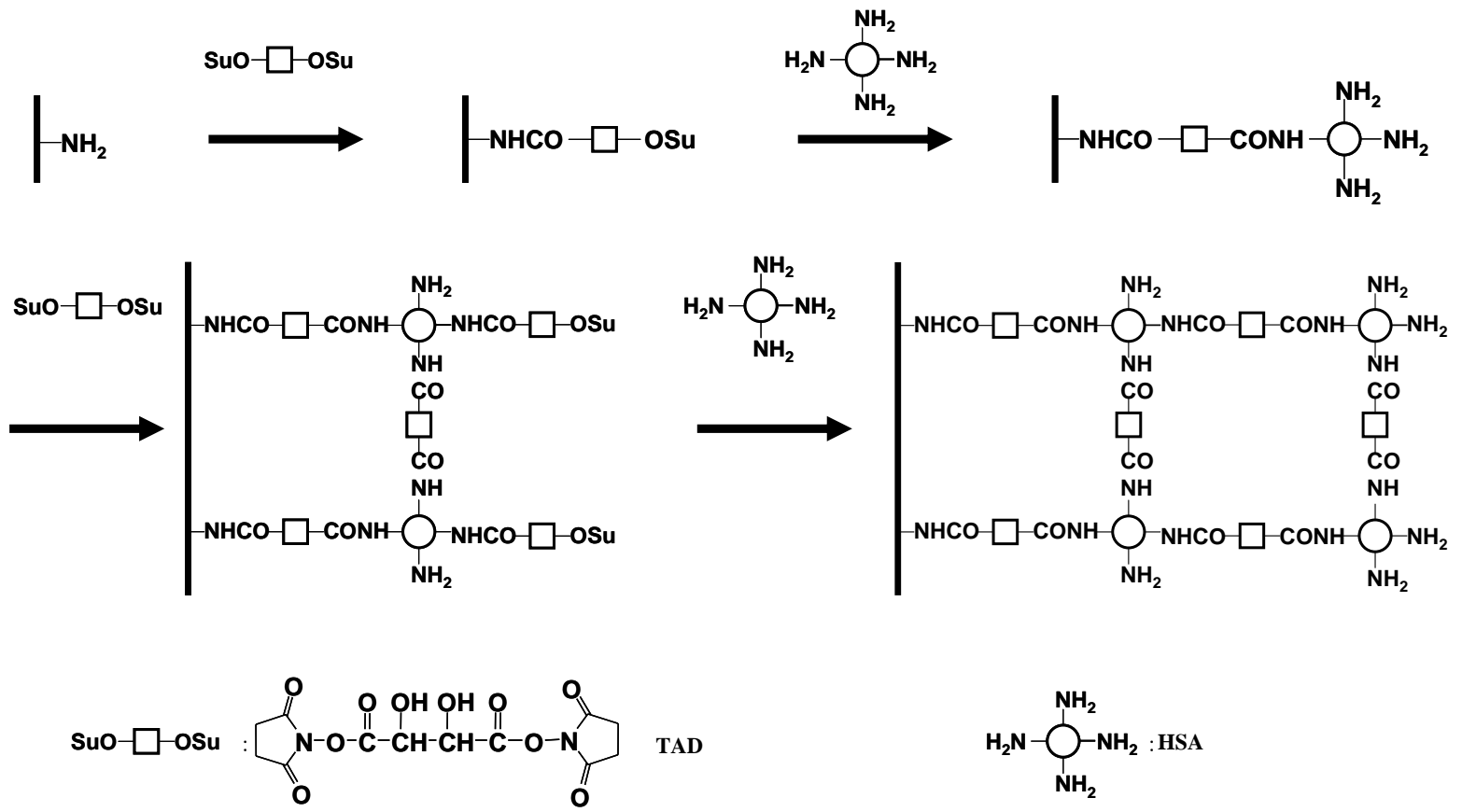

TAD

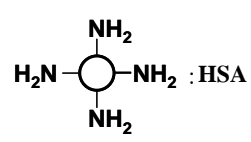

Fig. (2). Chemical scheme for immobilization of HSA on SUS316L by alternating immersion. 
formed by using an FT-IR 8400 S spectrometer (Shimazu Co., Kyoto, JAPAN) equipped with an ATR system (MIRacle A; PIKE Technologys, WI, USA). The nanoscale structures of the coated SUS316L samples were examined by AFM (SPA 400; Seiko Instruments Inc., Chiba, JAPAN) with a micro cantilever (SI-DF 20; Seiko Instruments Inc., Chiba, JAPAN) in the dynamic force mode (DFM). The images were analyzed by using Spisel 32 software (Seiko Instruments Inc., Chiba, JAPAN). To analyze their chemical compositions, the AS and HSA/TAD-20 samples were characterized by XPS (VG Theta Probe; Thermo Fisher Scientific, Inc., USA) using monochromatic Al Ka exciting radiation. The electron take-off angle was $37^{\circ}$ and XPS spectra over a range of binding energies (BEs) of $0-1400 \mathrm{eV}$ were acquired by using an analyzer pass energy of $300 \mathrm{eV}$ (survey scan) or $100 \mathrm{eV}$ (narrow scan).

\section{Test of the Stability of the Immobilized HSA/TAD Layer}

To examine the stability of the immobilized HSA/TAD layer on HSA/TAD-20, the surface-modified SUS316L was immersed in $1 \mathrm{M}$ aqueous $\mathrm{NaCl}$ or $5 \mathrm{vol} \%$ aqueous SDS for 10 min Samples were then rinsed adequately with distilled water and dried with nitrogen gas. The surface of the samples before and after washing was analyzed by using ATRFTIR.

\section{In Vitro Blood Compatibility Test}

The blood compatibility of the coated samples was tested by using murine whole blood. Samples of the bare metal, AS, and HSA/TAD-10 were immersed in whole mouse blood for 5 or $30 \mathrm{~min}$, washed with $0.1 \mathrm{M}$ phosphatebuffered solution ( $\mathrm{pH} 7.4)$, and fixed by immersion in $10 \%$ formalin neutral buffer solution ( $\mathrm{pH}$ 7.4) for $24 \mathrm{~h}$. The formalin-fixed samples were dehydrated with ethanol and tertbutanol, and then lyophilized. The surface of the lyophilized samples was examined by scanning electron microscopy (SEM) (SEMEDX II type NS; JEOL Ltd., Tokyo, JAPAN).

\section{RESULTS AND DISCUSSION}

\section{Surface Characterization}

The dependence of the water contact angle of the sample surface on the number of cycles of alternating immersion in TAD/DMSO and $\mathrm{HSA} / \mathrm{H}_{2} \mathrm{O}$ solutions is shown in Fig. (3). The contact angle decreased from $73.6^{\circ}$ to $66.5^{\circ}$ within five cycles. After more than five cycles, the contact angle increased slightly. These results suggested that hydrophilic HSA was immobilized and completely covered on the surface of AS within five cycles of alternating immersion in HSA and TAD solutions. The slight increase in the contact angle after five cycles could be due to an increase in the density of crosslinks in the HSA/TAD layer as a result of the reaction between HSA and the active ester groups remaining in the HSA/TAD layer.

To confirm the immobilization of the HSA/TAD layer on the bare metal surfaces, ATR-FTIR spectra of the bare metal, AS, HSA/TAD samples were recorded, and the results are shown in Fig. (4). The ATR-FTIR spectra of the bare metal and AS samples showed a band at around $1120 \mathrm{~cm}^{-1}$, assigned to metallic oxide. In contrast, HSA/TAD samples showed a peak in the ATR-FTIR spectra at around $1650 \mathrm{~cm}^{-1}$, which we assigned to the amide I band corresponding to peptide bonds in the HSA molecules. The ratio of intensity of the amide I band $\left(1650 \mathrm{~cm}^{-1}\right)$ to that of the metallic oxide band $\left(1118 \mathrm{~cm}^{-1}\right)\left(\mathrm{T}_{1650} / \mathrm{T}_{1118}\right)$ clearly increased with increasing numbers of alternating HSA/TAD immersion cycles, as ashown in Table 1, providing evidence that HSA molecules

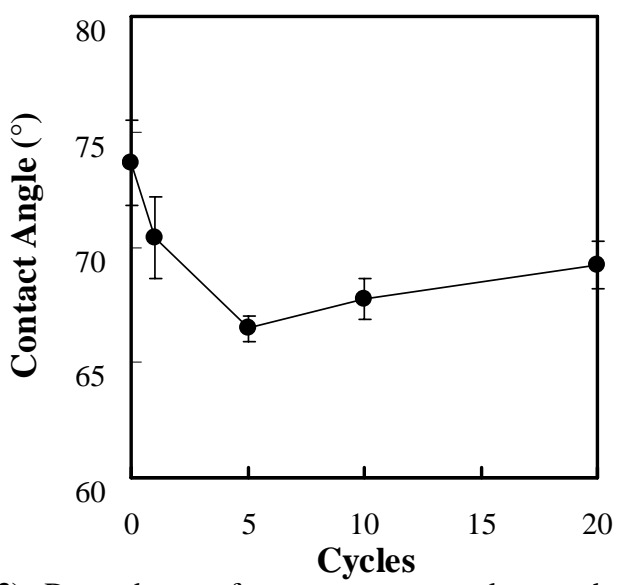

Fig. (3). Dependence of water contact angles on the number of cycles of alternating immersion of the resulting samples.

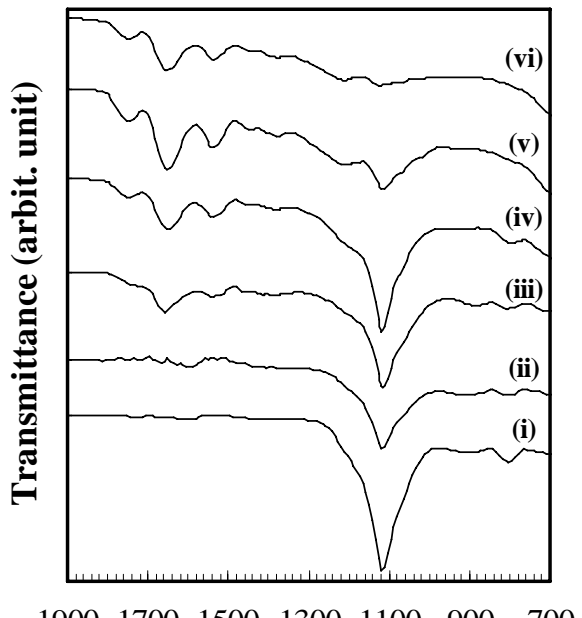

Wavenumber $\left(\mathrm{cm}^{-1}\right)$

Fig. (4). ATR-FTIR spectra of the bare metal (i), AS (ii), and the HSA/TAD samples: HSA/TAD-1 (iii), HSA/TAD-5 (iv), HSA/TAD-10 (v), and HSA/TAD-20 (vi).

Table 1. Intensity Ratios of the Amide I Band at Around $1650 \mathrm{~cm}^{-1}\left(T_{1650}\right)$ and the Metallic Oxide Band at $1118 \mathrm{~cm}^{-1}\left(T_{1118}\right)$ in ATR-FTIR Spectra of SUS316L Samples

\begin{tabular}{|c|c|}
\hline Sample & $\begin{array}{c}\text { Intensity Ratio } \\
\left(\mathbf{T}_{\mathbf{1 6 5 0}} / \mathbf{T}_{\mathbf{1 1 1}}\right)\end{array}$ \\
\hline \hline Bare SUS316L & 0 \\
\hline Aminosilanized SUS316L & 0.40 \\
\hline HSA-TAD 1 cycle & 0.80 \\
\hline HSA-TAD 5 cycles & 0.81 \\
\hline HSA-TAD 10 cycles & 0.92 \\
\hline HSA-TAD 20 cycles & 1.09 \\
\hline
\end{tabular}


were covalently immobilized on the surface of SUS316L by alternating immersion in HSA and TAD solutions.

In order to confirm the presence of covalent bonding, the surface morphology of the treated SUS316L samples was examined by AFM, as shown in Fig. (5). The surface of the bare metal was smooth (Fig. 5a), whereas that of AS was bumpy owing to the polymerization of $\gamma$-APS (Fig. 5b). The HSA/TAD-coated samples had markedly roughened surfaces, the roughness of which was related to the number of cycles of alternating immersion in HSA and TAD solutions (Fig. 5c-f). These results confirmed that the SUS316L surfaces were covalently modified by the globular protein HSA crosslinked by TAD.

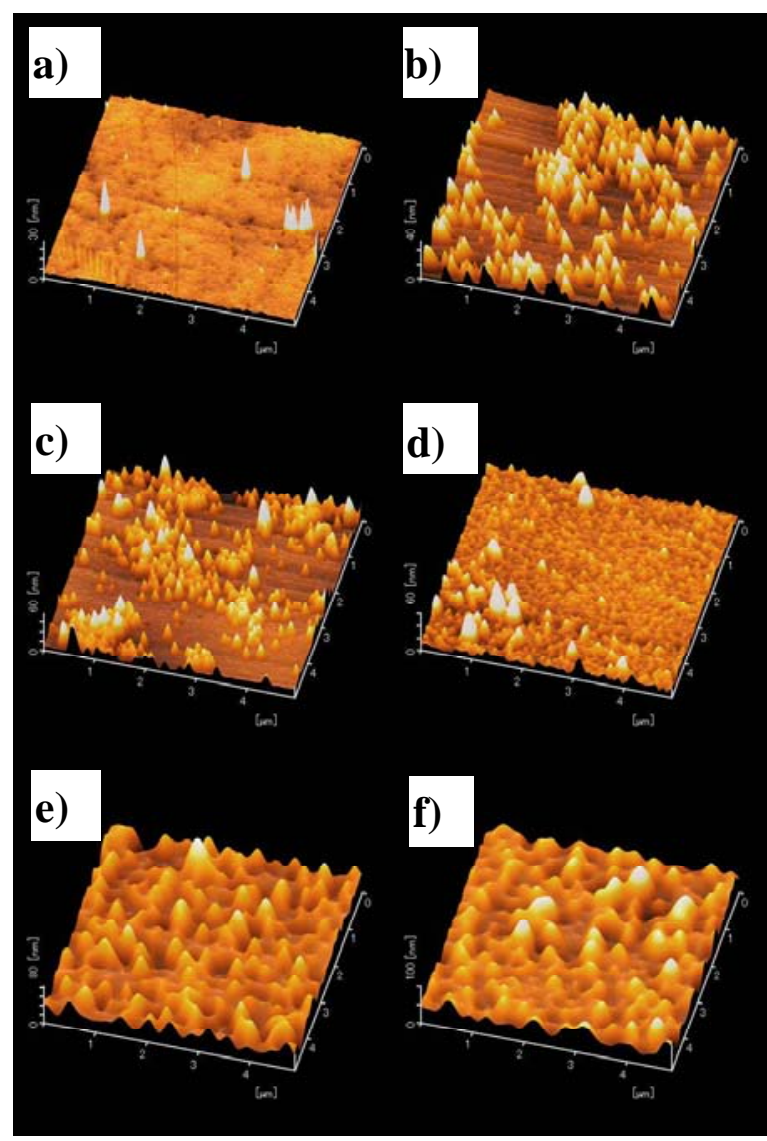

Fig. (5). AFM images of the surface of the bare metal (a), AS (b), and the HSA/TAD samples: HSA/TAD-1 (c), HSA/TAD-5 (d), HSA/TAD-10 (e), and HSA/TAD-20 (f).

The XPS scan of the SUS316L samples surface is shown in Fig. (6). The XPS scan of the AS surface shows the presence of $\mathrm{Fe}(2 p 3)$ and $\mathrm{Cr}(2 p 3)$ peaks assigned to metal oxide, and $\mathrm{O}(1 s), \mathrm{N}(1 s)$ and $\mathrm{C}(2 s)$ peaks assigned to $\gamma$-APS layer or hydrocarbon (Fig. 6i). The presence of $\mathrm{Fe}(2 p 3)$ and $\mathrm{Cr}$ ( $2 p 3)$ peaks indicates that a thin layer of $\gamma$-APS was formed on the surface of SUS316L. On the other hand, no metal oxide peaks were observed on the surface of HSA/TAD-20, as shown in Fig. (6ii), indicating that a thick HSA/TAD layer was formed by alternating immersion in HSA and TAD solutions.

\section{Stability Testing of the Immobilized HSA/TAD Layer}

To demonstrate the presence of a covalently immobilized HSA/TAD layer, HSA/TAD-20 was washed with $1 \mathrm{M}$ aque- ous $\mathrm{NaCl}$ or 5 vol\% aqueous SDS. If the HSA/TAD layer had been immobilized by physicochemical adsorption, with electrostatic or hydrophobic interactions, the HSA/TAD layer would have been eliminated by washing with either of these solutions. ATR-FTIR spectra of the HSA/TAD-20 before and after washing by the two solution are shown in Fig. (7). When HSA/TAD-20 was washed with $1 \mathrm{M} \mathrm{NaCl}$, no significant decrease in the intensity of the amide I band at around $1650 \mathrm{~cm}^{-1}$ was observed, as shown in Fig. (7i). Similarly, the amide I band at around $1650 \mathrm{~cm}^{-1}$ remained after washing with 5 vol\% SDS, as shown in Fig. (7ii). These results confirm that the HSA/TAD layer was covalently immobilized on the surface of SUS316L and that it was stable even in an environment where hydrophobic and electrostatic interactions were reduced.

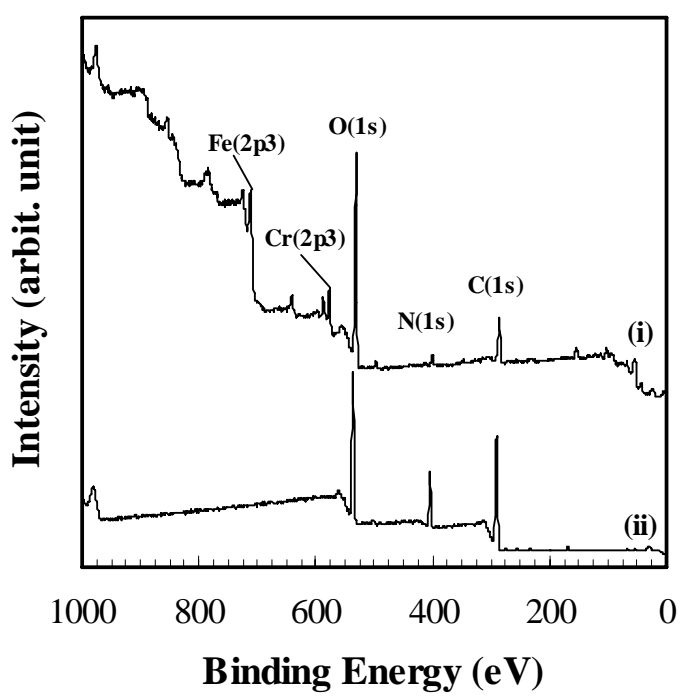

Fig. (6). XPS spectra of the AS (i) and HSA/TAD-20 (ii) samples.

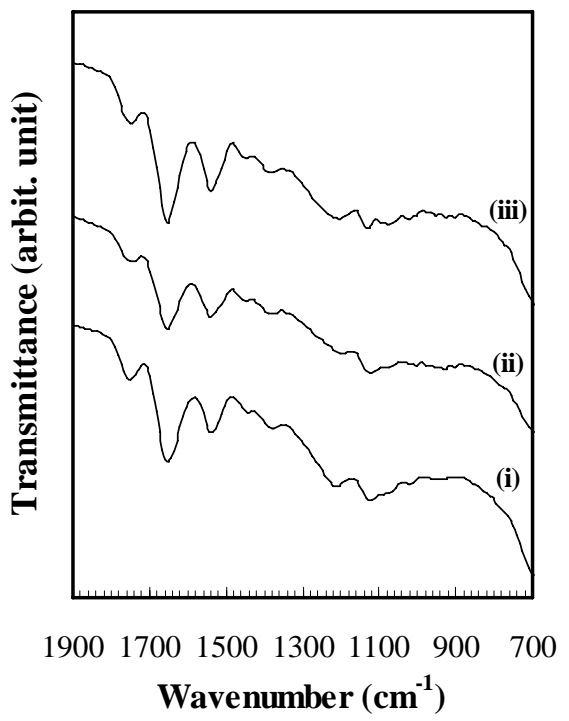

Fig. (7). ATR-FTIR spectra of HSA/TAD-20 before and after washing in $1 \mathrm{M} \mathrm{NaCl}$ and 5 vol\% SDS aqueous solutions: (i) before washing, (ii) washed with $1 \mathrm{M} \mathrm{NaCl}$, (iii) washed with 5 vol\% SDS.

\section{In Vitro Blood Compatibility}

The in vitro blood compatibility of the bare metal, AS, and HSA/TAD-10 samples was examined to evaluate the 
thrombus-formation activity of the HSA/TAD layer. Although thrombus formation occurred on each sample after 5 and $30 \mathrm{~min}$, the amount of fibrin fiber on the samples increased in the order AS > bare metal > HSA/TAD-10. Namely, more fibrin fiber was formed on the AS surface than on the bare metal surface, because AS introduced amino groups onto the surface. This produced a positive charge causing blood platelet and protein to adsorb onto the AS surface, resulting in ready formation of thrombus. On the other hand, thrombus was scarcely formed on the HSA/TAD-10 surface after 5 or $30 \mathrm{~min}$. It is known that albumin immobilized on various kinds of materials has anti-thrombogenic properties as a result of the negative charge of the protein $[18,19]$. In our case, the amino groups of HSA were partially converted into carboxylic groups by TAD when one active ester group of TAD reacted with an amino group of HSA and the other was hydrolyzed. We therefore suppose that the anti-thrombogenicity of HSA/TAD-10 is a result of its negatively charged surface.
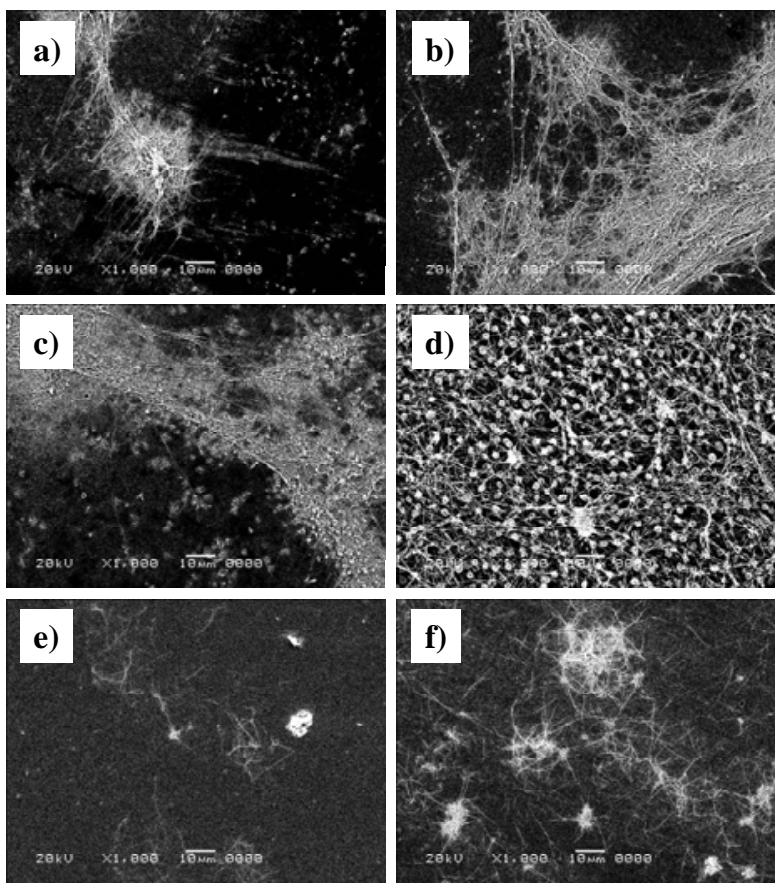

Fig. (8). Thrombus formation on the bare metal (a, b), AS (c, d), and HSA/TAD-10 (e, f) samples. Immersion times: $5 \mathrm{~min}(\mathbf{a}, \mathbf{c}$, and e), and $30 \min (\mathbf{b}, \mathbf{d}$, and $\mathbf{f})$.

\section{CONCLUSION}

The surface of aminosilanized SUS316L was covalently modified by alternating immersion in solutions of HSA and TAD. The thickness of the HSA/TAD layer increased with increasing number of cycles of alternating immersion in the two solutions. The immobilization of the HSA/TAD layer through covalent bonding, rather than by electrostatic or hydrophobic interactions, was confirmed by immersing the surface-modified SUS316L in solutions of a surfactant and of a salt. In addition, the HSA/TAD layer on the SUS316L surface showed anti-thrombogenic properties. These results suggested that alternating immersion of aminosilanized
SUS316L in HSA and TAD solutions is a simple method for immobilization of the biopolymer on metallic materials. This immobilization technique is expected to be useful for intravascular devices such as coronary stents.

\section{ACKNOWLEDGEMENT}

This work was financially supported in part by the research program on development of innovative technology of the Japan Science and Technology Agency.

\section{REFERENCES}

[1] Brunski JB. Metals, "Biomaterials science", Ratner BD, Hoffman AS, Schoen FJ (Ed), Elsevier academic press, California, 2004; p. 137.

[2] Hanson SR. Blood coagulation and blood materials interactions, "Biomaterials science", Ratner BD, Hoffman AS, Schoen FJ (Ed), Elsevier academic press, California, 2004; p. 332.

[3] Tan Q, Ji J, Barbosa MA, et al. Constructing thromboresistant surface on biomedical stainless steel via layer-by-layer deposition anticoagulant. Biomaterials 2003; 24: 4699-705.

[4] Cai K, Rechtenbach A, Hao J, et al. Polysaccharide-protein surface modification of titanium via a layer-by-layer technique: Characterization and cell behaviour aspects. Biomaterials 2005; 26: 5960-71.

[5] Brynda E, Houska M, Jiroušková M, et al. Albumin and heparin multilayer coatings for blood-contacting medical devices. J Biomed Mater Res 2000; 51: 249-57

[6] Puleo DA. Retention of enzymatic activity immobilized on silanized Co-Cr-Mo and Ti-6Al-4V. J Biomed Mater Res 1997; 37: 222-8.

[7] Nanci A, Wuest JD, Peru L, et al. Chemical modification of titanium surfaces for covalent attachment of biological molecules. J Biomed Mater Res 1998; 40: 324-35.

[8] Pitt WG, Morris RN, Mason ML, et al. Attachment of hyaluronan to metallic surfaces. J Biomed Mater Res 2004; 68A: 95-106.

[9] Müller R, Abke J, Schnell E, et al. Influence of surface pretreatment of titanium- and cobalt-based biomaterials on covalent immobilization of fibrillar collagen. Biomaterials 2006; 27: 4059-68.

[10] Yoshioka T, Tsuru K, Hayakawa S, et al. Preparation of alginic acid layers on stainless-steel substrates for biomedical applications. Biomaterials 2003; 24: 2889-94.

[11] Müller R, Abke J, Schnell E, et al. Surface engineering of stainless steel materials by covalent collagen immobilization to improve implant biocompatibility. Biomaterials 2005; 26: 6962-72.

[12] Saito H, Taguchi T, Kobayashi H, et al. Physicochemical properties of gelatin gels prepared using citric acid derivative. Mater Sci Eng C 2004; 24: 781-5.

[13] Aoki H, Taguchi T, Saito H, et al. Rheological evaluation of gelatin gels prepared with a citric acid derivative as a novel crosslinker. Mater Sci Eng C 2004; 24: 787-90.

[14] Saito H, Taguchi T, Aoki $\mathrm{H}$, et al. $\mathrm{pH}$-responsive crosslinkers swelling behavior of collagen gels prepared by novel based on naturally derived di- or tricarboxylic acids. Acta Biomater 2007; 3 : 89-94.

[15] Kakinoki S, Taguchi T, Saito $\mathrm{H}$, et al. Injectable in situ forming drug delivery system for cancer chemotherapy using a novel tissue adhesive: Characterization and in vitro evaluation. Eur $\mathrm{J}$ Pharm Biopharm 2007; 66: 383-390.

[16] Taguchi T, Saito H, Uchida Y, et al. Bonding of soft tissues using a novel acid derivative tissue adhesive consisting of a citric and collagen. Mater Sci Eng C 2004; 24: 775-80.

[17] Taguchi T, Saito H, Iwasashi M, et al. Development of a novel glue consisting of naturally-derived biomolecules: Citric acid and human serum albumin. J Nanosci Nanotechnol 2007; 7: 742-7.

[18] Ishikawa Y, Sasakawa S, Takase M, et al. Effect of albumin immobilization by plasma polymerization on platelet reactivity. Thromb Res 1984; 35: 193-202.

[19] Kamath KR, Park K. Surface modification of polymeric biomaterials by albumin grafting using gamma-irradiation. J Appl Biomat 1994; 5: 163-73. 